\title{
Multiple stability and instability of Cohen-Grossberg neural network with unbounded time-varying delays
}

\author{
Yanheng Chen ${ }^{1,2^{*}}$ and Songfang Jia
}

\author{
"Correspondence: \\ yanheng_1980@sina.com \\ ${ }^{1}$ Key Laboratory for Nonlinear \\ Science and System Structure, and \\ School of Mathematic and Statistics, \\ Chongqing Three Gorges University, \\ Chongqing, China \\ ${ }^{2}$ Key Laboratory of Intelligent \\ Information Processing and Control \\ of Chongqing Municipal Institutions \\ of Higher Education, Chongqing \\ Three Gorges University, \\ Chongqing, China
}

\begin{abstract}
In this paper, multiple stability and instability of Cohen-Grossberg neural network with unbounded time-varying delays are studied. Based on the geometrical configuration of activation functions and some rigorous mathematical analysis, some algebraic criteria are proposed to guarantee coexistence of multiple stable equilibrium points and multiple unstable equilibrium points in the model. Moreover, using the partition space method, we prove that the discussed model has at least $3^{n}$ equilibrium points, $2^{n}$ of them are locally $\mu$-stable and others are unstable. Finally, the numerical example and its simulation show the effectiveness of the proposed results.
\end{abstract}

Keywords: Cohen-Grossberg neural network; Multiple stability and instability; Unbounded time-varying delays

\section{Introduction}

The Cohen-Grossberg neural network model, proposed by Cohen and Grossberg in 1983 [1], has been attracting much attention because of its wide application in various engineering fields and because of it being highly inclusive of other neural networks such as Hopfield neural network, cellular neural network, recurrent neural network, and so on. Hence many scholars devoted themselves to the research of this aspect (see [2-18]). In some practical applications and hardware implementations of artificial neural networks, time delays are inevitable due to the finite switching speed of the amplifiers and the interneurons conduction distances, and they are even time-varying and unbounded in some cases such as the memory activation function of the human brain neural network model. Therefore, it is more suitable to introduce unbounded time-varying delays to the neural network, especially to Cohen-Grossberg models, and some results have been reported recently, for example, [19-28].

In the applications of pattern recognition, the addressable memories of patterns are stored as stable equilibrium points. Thus it is necessary that there exist multiple stable equilibrium points for neural networks. The coexistence of multiple equilibrium points and their local stability, which is usually referred to as the multistability of neural network models, has been reported in depth in the last years (see [29-43] and the references therein). Wang et al. in [35] studied a class of neural networks with $r$-level piecewise linear nondecreasing activation functions and showed that the $n$-neuron dynamical system

(c) The Author(s) 2019. This article is distributed under the terms of the Creative Commons Attribution 4.0 International License (http://creativecommons.org/licenses/by/4.0/), which permits unrestricted use, distribution, and reproduction in any medium, provided you give appropriate credit to the original author(s) and the source, provide a link to the Creative Commons license, and indicate if changes were made. 
had exact $(2 r+1)^{n}$ equilibrium points, of which $(r+1)^{n}$ were locally exponentially stable and the others were unstable. By using the partition space method, [41] proved that neural networks with unbounded time-varying delays could exhibit at least $3^{n}$ equilibrium points, $2^{n}$ of them are locally $\mu$-stable and others are unstable. In [43], based on the geometrical configuration of activation functions and mathematic tools, some novel algebraic criteria were proposed to guarantee the coexistence of $25^{n}$ equilibrium points, in which $9^{n}$ equilibrium points are locally $\mu$-stable, for the memristor-based complex-valued neural networks with non-monotonic piecewise nonlinear activation functions and unbounded time-varying delays. From the references mentioned above, we find that the multistability of Cohen-Grossberg neural networks with unbounded time-varying delays is a challenging problem.

Motivated by the challenging problem, we investigate the multistability of a CohenGrossberg neural network with unbounded time-varying delay and nondecreasing activation functions in this paper and prove that the considered model has $3^{n}$ equilibrium points, and $2^{n}$ of them are locally $\mu$-stable, the remaining ones are unstable. Compared with the literature [41], the results are more general. The rest of this paper is organized as follows. In Sect. 2, the Cohen-Grossberg model and some preliminaries are given. The main results are presented and proved in Sect. 3. The corollaries and comparison with the results of existing literature are presented in Sect. 4. A numerical example with its simulation is showed in Sect. 5 to illustrate the effectiveness the proposed results. Finally, conclusions are drawn in Sect. 6.

\section{Preliminaries}

In this paper, the following Cohen-Grossberg neural network is considered.

$$
\frac{\mathrm{d} x_{i}(t)}{\mathrm{d} t}=-a_{i}\left(x_{i}(t)\right)\left[b_{i}\left(x_{i}(t)\right)-\sum_{j=1}^{n} c_{i j} g_{j}\left(x_{j}(t)\right)-\sum_{j=1}^{n} d_{i j} f_{j}\left(x_{j}(t-\tau(t))\right)+I_{i}\right], \quad t \geq 0, \quad(1)
$$

where $i=1,2, \ldots, n, x_{i}(t)$ denotes the state variable associated with the $i$ th neuron at time $t ; a_{i}\left(x_{i}(t)\right)$ represents an amplification function at time $t ; b_{i}\left(x_{i}(t)\right)$ is an appropriate inhibition behavior function at time $t$ such that the solutions of model (1) remain bounded; $g_{j}\left(x_{j}(t)\right)$ and $f_{j}\left(x_{j}(t-\tau(t))\right)$ denote the activation functions of the $j$ th neuron unit at time $t$ without and with time delays, respectively, and $C=\left(c_{i j}\right)_{n \times n}$ and $D=\left(d_{i j}\right)_{n \times n}$ are the corresponding connection weights matrices; $\tau(t)$ corresponds to the transmission delay and satisfies $\tau(t) \geq 0 ; I_{i}$ is the constant external input of the network on the $i$ th neuron.

The initial conditions of model (1) are assumed to be $x_{i}(s)=\varphi_{i}(s), s \leq 0, i=1,2, \ldots, n$, where $\varphi_{i}(s)$ is a real-valued continuous function bounded on $(-\infty, 0]$, except that finite points existing at the left and right limits are continuous to the right. Throughout this paper, we make the following assumptions.

(H1) For each $i \in 1,2, \ldots, n$, the amplification function $a_{i}(u)$ is nonnegative continuous and satisfies

$$
0<\underline{a}_{i} \leq a_{i}(u) \leq \bar{a}_{i}<\infty, \quad u \in R, i=1,2, \ldots, n .
$$

And let two $n$-dimensional positive diagonal matrices $\hat{A}=\operatorname{diag}\left\{\bar{a}_{1}, \bar{a}_{2}, \ldots, \bar{a}_{n}\right\}$ and $\check{A}=\operatorname{diag}\left\{\underline{a}_{1}, \underline{a}_{2}, \ldots, \underline{a}_{n}\right\}$. 
(H2) $b_{i}(u)$ is an odd function and monotone increasing, and there exists an $n$-dimensional positive diagonal matrix $B=\operatorname{diag}\left\{b_{1}, b_{2}, \ldots, b_{n}\right\}$ such that

$$
\frac{b_{i}(u)-b_{i}(v)}{u-v} \geq b_{i}, \quad u, v \in R, u \neq v, i=1,2, \ldots, n .
$$

(H3) $g_{j}(\cdot)$ and $f_{j}(\cdot)$ are nondecreasing sigmoid continuous nonlinear function or nondecreasing piecewise continuous linear function, and there exist constants $p_{j} \leq q_{j}, m_{j} \leq M_{j}, m_{j}^{\prime} \leq M_{j}^{\prime}, m_{j}^{\prime \prime} \leq M_{j}^{\prime \prime}$, so that

$$
\begin{aligned}
& m_{j}^{\prime}=\lim _{x \rightarrow-\infty} g_{j}(x), \quad M_{j}^{\prime}=\lim _{x \rightarrow+\infty} g_{j}(x), \\
& m_{j}^{\prime \prime}=\lim _{x \rightarrow-\infty} f_{j}(x), \quad M_{j}^{\prime \prime}=\lim _{x \rightarrow+\infty} f_{j}(x), \\
& 0 \leq \underline{\sigma}_{j}^{l} \leq \frac{g_{j}(u)-g_{j}(v)}{u-v} \leq \bar{\sigma}_{j}^{l}, \quad 0 \leq \underline{\delta}_{j}^{l} \leq \frac{f_{j}(u)-f_{j}(v)}{u-v} \leq \bar{\delta}_{j}^{l}, \quad \forall u, v \in\left(-\infty, p_{j}\right), \\
& 0 \leq \underline{\sigma}_{j}^{m} \leq \frac{g_{j}(u)-g_{j}(v)}{u-v} \leq \bar{\sigma}_{j}^{m}, \quad 0 \leq \underline{\delta}_{j}^{m} \leq \frac{f_{j}(u)-f_{j}(v)}{u-v} \leq \bar{\delta}_{j}^{m}, \quad \forall u, v \in\left[p_{j}, q_{j}\right], \\
& 0 \leq \underline{\sigma}_{i}^{r} \leq \frac{g_{j}(u)-g_{j}(v)}{u-v} \leq \bar{\sigma}_{j}^{r}, \quad 0 \leq \underline{\delta}_{j}^{r} \leq \frac{f_{j}(u)-f_{j}(v)}{u-v} \leq \bar{\delta}_{j}^{r}, \quad \forall u, v \in\left(p_{j},+\infty\right),
\end{aligned}
$$

where $m_{j}=\min \left\{m_{j}^{\prime}, m_{j}^{\prime \prime}\right\}, M_{j}=\min \left\{M_{j}^{\prime}, M_{j}^{\prime \prime}\right\}, \bar{\sigma}_{j}=\max \left\{\bar{\sigma}_{j}^{l}, \bar{\sigma}_{j}^{m}, \bar{\sigma}_{j}^{r}\right\}$, $\bar{\delta}_{j}=\max \left\{\bar{\delta}_{j}^{l}, \bar{\delta}_{j}^{m}, \bar{\delta}_{j}^{r}\right\}, j=1,2, \ldots, n$, and define two $n$-dimensional positive diagonal matrices $\Sigma^{g}=\operatorname{diag}\left\{\bar{\sigma}_{1}, \bar{\sigma}_{2}, \ldots, \bar{\sigma}_{n}\right\}$ and $\Delta^{f}=\operatorname{diag}\left\{\bar{\delta}_{1}, \bar{\delta}_{2}, \ldots, \bar{\delta}_{n}\right\}$. The superscripts "l", " $m$ ", " $r$ " denote "left", "middle", and "right", respectively.

It is not hard to find such activation functions as the sigmoid continuous nonlinear function $f(x)=\tanh (x)=\frac{e^{x}-e^{-x}}{e^{x}+e^{-x}}$, piecewise continuous linear function $g(x)=\frac{|x+1|-|x+1|}{2}$, which are different functions, but the properties of the functions can be discussed by common interval separation points. Based on the geometric structure of the activation function, we can define the interval of a one-dimensional real number space as follows:

$$
(-\infty,+\infty)=\left(-\infty, p_{i}\right) \cup\left[p_{i}, q_{i}\right] \cup\left(q_{i},+\infty\right), \quad i=1,2, \ldots, n,
$$

then the $n$-dimensional real number space $R^{n}$ can be divided into $3^{n}$ non-intersection subregions. For convenience, let $\Phi$ denote the set of these subregions, and so

$$
\Phi=\left\{\prod_{i=1}^{n} w_{i} \mid w_{i}=\left(-\infty, p_{i}\right),\left[p_{i}, q_{i}\right] \text { or }\left(q_{i},+\infty\right)\right\} .
$$

For each $\prod_{i=1}^{n} w_{i} \in \Phi$, we define the following index subsets with respect to different interval as $N_{1}=\left\{i \mid w_{i}=\left(-\infty, p_{i}\right), i=1,2, \ldots, n\right\}, N_{2}=\left\{i \mid w_{i}=\left[p_{i}, q_{i}\right], i=1,2, \ldots, n\right\}, N_{3}=\{i \mid$ $\left.w_{i}=\left(q_{i},+\infty\right), i=1,2, \ldots, n\right\}$.

Furthermore, $\Phi$ can be separated into two parts: $\Phi_{1}=\left\{\prod_{i=1}^{n} w_{i} \mid w_{i}=\left(-\infty, p_{i}\right)\right.$ or $\left(q_{i}\right.$, $+\infty)\}, \Phi_{2}=\Phi-\Phi_{1}$. Obviously, $\Phi_{1}$ is composed of $2^{n}$ subregions and $\Phi_{2}$ contains $3^{n}-2^{n}$ subregions. 
To facilitate showing the existence of equilibrium points of model (1), we define new sets

$$
\begin{aligned}
& \Omega=\left\{\prod_{i=1}^{n} v_{i} \mid v_{i}=\left[-E_{i}, p_{i}\right],\left[p_{i}, q_{i}\right] \text { or }\left[q_{i}, E_{i}\right]\right\}, \\
& \Omega_{1}=\left\{\prod_{i=1}^{n} v_{i} \mid v_{i}=\left[-E_{i}, p_{i}\right] \text { or }\left[q_{i}, E_{i}\right]\right\},
\end{aligned}
$$

where $E_{i}=2 b_{i}^{-1}\left[\sum_{j=1}^{n}\left(\left|c_{i j}\right|+\left|d_{i j}\right|\right) \max \left\{m_{j}, M_{j}\right\}+\left|I_{i}\right|+\max \left\{\left|b_{i}\left(p_{i}\right)\right|, \mid b_{i}\left(q_{i} \mid\right\}\right], i=1,2, \ldots, n\right.$.

For each $\prod_{i=1}^{n} v_{i} \in \Omega$, we can similarly define its index subsets: $N_{1}^{\prime}=\left\{i \mid v_{i}=\left[-E_{i}, p_{i}\right], i=\right.$ $1,2, \ldots, n\}, N_{2}^{\prime}=\left\{i \mid v_{i}=\left[p_{i}, q_{i}\right], i=1,2, \ldots, n\right\}, N_{3}^{\prime}=\left\{i \mid v_{i}=\left[q_{i}, E_{i}\right], i=1,2, \ldots, n\right\}$.

\section{Main results}

Because an equilibrium point of system (1) is a constant satisfying the equation $b_{i}\left(x_{i}(t)\right)-$ $\sum_{j=1}^{n} c_{i j} g_{j}\left(x_{j}(t)\right)-\sum_{j=1}^{n} d_{i j} f_{j}\left(x_{j}(t)\right)+I_{i}=0$, it is obvious that of model (1) has the same equilibrium point with the following system:

$$
\frac{\mathrm{d} x_{i}(t)}{\mathrm{d} t}=-a_{i}\left(x_{i}(t)\right)\left[b_{i}\left(x_{i}(t)\right)-\sum_{j=1}^{n} c_{i j} g_{j}\left(x_{j}(t)\right)-\sum_{j=1}^{n} d_{i j} f_{j}\left(x_{j}(t)+I_{i}\right], \quad t \geq 0,\right.
$$

for $i=1,2, \ldots, n$. Therefore, we can investigate the existence of multiple equilibrium points of model (2) instead of (1).

Theorem 1 For any $\prod_{i=1}^{n} w_{i} \in \Phi$, if

$$
\left\{\begin{array}{l}
-b_{i}\left(p_{i}\right)+c_{i i} g_{i}\left(p_{i}\right)+d_{i j} f_{i}\left(p_{i}\right)+\sum_{\substack{j=1 \\
j \neq i}}^{n} \max \left\{\left(c_{i j}+d_{i j}\right) m_{j},\left(c_{i j}+d_{i j}\right) M_{j}\right\}-I_{i}<0, \\
\quad i \in N_{1} \cup N_{2}, \\
-b_{i}\left(q_{i}\right)+c_{i i} g_{i}\left(q_{i}\right)+d_{i i} f_{i}\left(q_{i}\right)+\sum_{\substack{j=1 \\
j \neq i}}^{n} \min \left\{\left(c_{i j}+d_{i j}\right) m_{j},\left(c_{i j}+d_{i j}\right) M_{j}\right\}-I_{i}>0, \\
\quad i \in N_{2} \cup N_{3},
\end{array}\right.
$$

where $N_{i} \cap N_{j}=\phi, i \neq j, i, j=1,2,3, N_{1} \cup N_{2} \cup N_{3}=\{1,2, \ldots, n\}$, then there is at least one equilibrium point of model (1) in $\prod_{i=1}^{n} w_{i}$.

Proof Let $\left(x_{1}, x_{2}, \ldots, x_{n}\right)$ be a point of $\prod_{i=1}^{n} v_{i} \in \Omega$. Then, for the $i$ th component $x_{i}$, fixing other components $x_{1}, \ldots, x_{i-1}, x_{i+1}, \ldots, x_{n}$, we can define a function as follows:

$$
F_{i}(u)=-b_{i}(u)+c_{i i} g_{i}(u)+d_{i i} f_{i}(u)+\sum_{\substack{j=1 \\ j \neq i}}^{n} c_{i j} g_{j}\left(x_{j}\right)+\sum_{\substack{j=1 \\ j \neq i}}^{n} d_{i j} f_{j}\left(x_{j}\right)-I_{i},
$$

where $i=1,2, \ldots, n$. Then, by (3), we can deduce the following:

(1) The function $F_{i}(u)$ is continuous in the interval $\left[-E_{i}, p_{i}\right]$, and $F_{i}\left(-E_{i}\right)>0, F_{i}\left(p_{i}\right)<0$. Then by the zero point theorem, there exists at least a point $\bar{x}_{i} \in\left(-E_{i}, p_{i}\right)$ such that $F_{i}\left(\bar{x}_{i}\right)=0$. On account of $a_{i}\left(x_{i}\right)>0$, by (H1), $\bar{x}_{i}$ is the equilibrium point of system (2) for the state component $x_{i}(t)$ of the $i$ th neuron in the interval $\left(-E_{i}, p_{i}\right)$. 
(2) If $u \in\left[p_{i}, q_{i}\right]$, then we have $F_{i}\left(p_{i}\right)>0, F_{i}\left(q_{i}\right)<0$. In view of $a_{i}\left(x_{i}\right)>0$, there exists at least a point $\bar{x}_{i} \in\left(p_{i}, q_{i}\right)$, which is the equilibrium point of system (2) for the state component $x_{i}(t)$ of the $i$ th neuron in the interval $\left(p_{i}, q_{i}\right)$.

(3) There exists at least a point $\bar{x}_{i} \in\left(q_{i}, E_{i}\right)$, which is the equilibrium point of system (2) for the state component $x_{i}(t)$ of the $i$ th neuron in the interval $\left(q_{i}, E_{i}\right)$ because of $F_{i}\left(q_{i}\right)>0, F_{i}\left(E_{i}\right)<0$, and $a_{i}\left(x_{i}\right)>0$.

Above all, the function $F_{i}(u)$ has at least one zero point in any subinterval. It follows that there is at least one equilibrium point of system (2) in $\prod_{i=1}^{n} v_{i} \in \Omega$.

In addition, we can define a mapping $\wp: \prod_{i=1}^{n} v_{i} \rightarrow \prod_{i=1}^{n} v_{i}$ for any given $x=\left(x_{1}, x_{2}, \ldots\right.$, $\left.x_{n}\right) \in \prod_{i=1}^{n} v_{i}$ such that $\wp\left(x_{1}, x_{2}, \ldots, x_{n}\right)=\left(\bar{x}_{1}, \bar{x}_{2}, \ldots, \bar{x}_{n}\right)$, where $\bar{x}_{i}$ is a solution of equation $F_{i}(u)=0, j=1,2, \ldots, n$. Since $f_{j}(\cdot), g_{j}(\cdot), j=1,2, \ldots, n$, are continuous by assumption (H3), the mapping $\wp$ is also continuous, then by Brouwer's fixed point theorem, there exists at least one fixed equilibrium point, denoted as $x^{\star}=\left(x_{1}^{\star}, x_{2}^{\star}, \ldots, x_{n}^{\star}\right)$, such that $\wp\left(x^{\star}\right)=x^{\star}$. Furthermore, components of the equilibrium point $x^{\star}$ satisfy $-E_{i}<x_{i}^{\star}<p_{i}$ for $i \in N_{1}^{\prime}$, $p_{i}<x_{i}^{\star}<q_{i}$ for $i \in N_{2}^{\prime}, q_{i}<x_{i}^{\star}<E_{i}$ for $i \in N_{3}^{\prime}$. It means that all the equilibrium points in $\prod_{i=1}^{n} v_{i}$ are located in its interior. In view of the arbitrariness of the region $\prod_{i=1}^{n} v_{i}$ and the sameness of the equilibrium points between model (1) and model (2), model (1) has at least $3^{n}$ equilibrium points in $\Omega$. From the definition of set $\Phi$ and $\Omega$, we know that the corresponding region of $\prod_{i=1}^{n} v_{i}$ is $\prod_{i=1}^{n} w_{i}$ and satisfies $\prod_{i=1}^{n} v_{i} \subseteq \prod_{i=1}^{n} w_{i}$. Hence it is easy to see that $x^{\star}$ is also an equilibrium of model (1) in $\prod_{i=1}^{n} w_{i}$, and so there is at least one equilibrium point of model (1) in $\prod_{i=1}^{n} w_{i}$.

For each equilibrium point $x^{\star}=\left(x_{1}^{\star}, \ldots, x_{n}^{\star}\right)$ of $\prod_{i=1}^{n} w_{i} \in \Phi_{1}$, we define its $\mu$-stability in $\prod_{i=1}^{n} w_{i}$ (local $\mu$-stability in $\Phi_{1}$ ), and prove the $\mu$-stability of all equilibrium points in $\Phi_{1}$ in the following Definition 1 and Theorem 2, respectively.

Definition 1 Let $\left(x_{1}(t), x_{2}(t), \ldots, x_{n}(t)\right)$ be an arbitrary solution of model (1) located in $\prod_{i=1}^{n} w_{i} \in \Phi_{1}$ with the initial state $x_{i}(s)=\varphi_{i}(s), s \in(-\infty, 0], i=1,2, \ldots, n$, and $\mu(t)$ be a nondecreasing function with $\mu(t) \rightarrow+\infty(t \rightarrow+\infty)$. Then $x^{\star}$ is said to be $\mu$-stable in $\prod_{i=1}^{n} w_{i}$ (locally $\mu$-stable in $\Phi_{1}$ ) if there is a positive constant $M$ such that

$$
\left|x_{i}(t)-x_{i}^{\star}\right| \leq \frac{M}{\mu(t)}
$$

Theorem 2 For any $\prod_{i=1}^{n} w_{i} \in \Phi_{1}$, given that

$$
\left\{\begin{array}{l}
-b_{i}\left(p_{i}\right)+c_{i i} g_{i}\left(p_{i}\right)+\sum_{\substack{j=1 \\
j \neq i}}^{n} \max \left\{c_{i j} m_{j}, c_{i j} M_{j}\right\}+\sum_{j=1}^{n} \max \left\{d_{i j} m_{j}, d_{i j} M_{j}\right\}-I_{i}<0, \\
i \in N_{1}, \\
-b_{i}\left(q_{i}\right)+c_{i i} g_{i}\left(q_{i}\right)+\sum_{\substack{j=1 \\
j \neq i}}^{n} \min \left\{c_{i j} m_{j}, c_{i j} M_{j}\right\}+\sum_{j=1}^{n} \min \left\{d_{i j} m_{j}, d_{i j} M_{j}\right\}-I_{i}>0, \\
i \in N_{3},
\end{array}\right.
$$

and the nondecreasing function $\mu(t)>0$ with

$$
\lim _{t \rightarrow+\infty} \mu(t)=+\infty, \quad 0 \leq \sup _{t \geq T^{*}} \frac{\dot{\mu}(t)}{\mu(t)} \leq \alpha, \quad \sup _{t \geq T^{*}} \frac{\mu(t)}{\mu(t-\tau(t))} \leq 1+\beta
$$


where $\alpha, \beta, T^{*}$ are nonnegative constants, $N_{1} \cap N_{3}=\phi, N_{1} \cup N_{3}=\{1,2, \ldots, n\}$. Then the equilibrium point $x^{\star}=\left(x_{1}^{\star}, \ldots, x_{n}^{\star}\right)$ is $\mu$-stable in $\prod_{i=1}^{n} w_{i}$ (locally $\mu$-stable in $\left.\Phi_{1}\right)$ if there are positive constants $\zeta_{1}, \zeta_{2}, \ldots, \zeta_{n}$ such that

$$
\begin{aligned}
& \left(-\underline{a}_{i} \beta_{i}+\alpha\right) \zeta_{i}+\sum_{j \in N_{1}} \zeta_{j} \bar{a}_{i} \bar{\sigma}_{j}^{l}\left|c_{i j}\right|+\sum_{j \in N_{3}} \zeta_{j} \bar{a}_{i} \bar{\sigma}_{j}^{r}\left|c_{i j}\right| \\
& +(1+\beta)\left(\sum_{j \in N_{1}} \zeta_{j} \bar{a}_{i} \bar{\delta}_{j}^{l}\left|d_{i j}\right|+\sum_{j \in N_{3}} \zeta_{j} \bar{a}_{i} \bar{\delta}_{j}^{r}\left|d_{i j}\right|\right)<0,
\end{aligned}
$$

where $i=1,2, \ldots, n$.

Proof From the comparison of (3) and (4), we know that there is at least one equilibrium point in $\prod_{i=1}^{n} w_{i}$ for model (1). The following proof will show that the equilibrium point is unique and $\mu$-stable in $\prod_{i=1}^{n} w_{i} \in \Phi_{1}$.

Let $x(t)=\left(x_{1}(t), x_{2}(t), \ldots, x_{n}(t)\right)$ be an arbitrary solution of model $(1)$ in $\prod_{i=1}^{n} v_{i} \in \Omega_{1}$ with the initial condition $x_{i}(s)=\varphi_{i}(s), s \in(-\infty, 0]$. We claim that $x(t)$ would remain in $\prod_{i=1}^{n} v_{i}$ for all $t \geq 0$.

For $x_{i}(t)$, suppose that $i \in N_{1}^{\prime}$. Then $i \in N_{1}$ on account of $\left[-E_{i}, p_{i}\right] \subset\left(-\infty, p_{i}\right]$. Further, by (4), one has $\varepsilon>0$ small enough such that

$$
-b_{i}\left(p_{i}-\varepsilon\right)+c_{i i} g_{i}\left(p_{i}-\varepsilon\right)+\sum_{\substack{j=1 \\ j \neq i}}^{n} \max \left\{c_{i j} m_{j}, c_{i j} M_{j}\right\}+\sum_{j=1}^{n} \max \left\{d_{i j} m_{j}, d_{i j} M_{j}\right\}-I_{i}<0 .
$$

And for $\varepsilon$ above, we can find some $t^{*} \geq 0$ such that $p_{i}-\varepsilon \leq x_{i}\left(t^{*}\right) \leq p_{i}$. It follows that

$$
\begin{aligned}
\left.\frac{\mathrm{d} x_{i}(t)}{\mathrm{d} t}\right|_{t=t^{*}}= & -a_{i}\left(x_{i}\left(t^{*}\right)\right)\left[b_{i}\left(x_{i}\left(t^{*}\right)\right)-\sum_{j=1}^{n} c_{i j} g_{j}\left(x_{j}\left(t^{*}\right)\right)-\sum_{j=1}^{n} d_{i j} f_{j}\left(x_{j}\left(t^{*}-\tau\left(t^{*}\right)\right)\right)+I_{i}\right] \\
\leq & a_{i}\left(x_{i}\left(t^{*}\right)\right)\left[-b_{i}\left(p_{i}-\varepsilon\right)\right. \\
& \left.+c_{i i} g_{i}\left(p_{i}-\varepsilon\right)+\sum_{\substack{j=1 \\
j \neq i}}^{n} \max \left\{c_{i j} m_{j}, c_{i j} M_{j}\right\}+\sum_{j=1}^{n} \max \left\{d_{i j} m_{j}, d_{i j} M_{j}\right\}-I_{i}\right] \\
< & 0 .
\end{aligned}
$$

On the other hand, we also can find some $t^{*} \geq 0$ such that $x_{i}\left(t^{*}\right)=-E_{i}$. By (H1), we get that

$$
\begin{aligned}
\left.\frac{\mathrm{d} x_{i}(t)}{\mathrm{d} t}\right|_{t=t^{*}} \geq & a_{i}\left(-E_{i}\right)\left[b_{i}\left(E_{i}\right)+c_{i i} g_{i}\left(-E_{i}\right)+\sum_{\substack{j=1 \\
j \neq i}}^{n} \min \left\{c_{i j} m_{j}, c_{i j} M_{j}\right\}\right. \\
& \left.+\sum_{j=1}^{n} \min \left\{d_{i j} m_{j}, d_{i j} M_{j}\right\}-I_{i}\right] \\
> & 0 .
\end{aligned}
$$


Similarly, for $x_{i}(t), i \in N_{3}^{\prime}$, there exists $\varepsilon>0$ small enough such that

$$
-b_{i}\left(q_{i}+\varepsilon\right)+c_{i i} g_{i}\left(q_{i}+\varepsilon\right)+\sum_{\substack{j=1 \\ j \neq i}}^{n} \min \left\{c_{i j} m_{j}, c_{i j} M_{j}\right\}+\sum_{j=1}^{n} \min \left\{d_{i j} m_{j}, d_{i j} M_{j}\right\}-I_{i}>0 .
$$

And for $\varepsilon$ above, we can discover some $t^{*} \geq 0$ such that $q_{i} \leq x_{i}\left(t^{*}\right) \leq q_{i}+\varepsilon$. It follows that

$$
\begin{aligned}
\left.\frac{\mathrm{d} x_{i}(t)}{\mathrm{d} t}\right|_{t=t^{*}} \geq & a_{i}\left(x_{i}\left(t^{*}\right)\right)\left[-b_{i}\left(q_{i}+\varepsilon\right)+c_{i i} g_{i}\left(q_{i}+\varepsilon\right)+\sum_{\substack{j=1 \\
j \neq i}}^{n} \min \left\{c_{i j} m_{j}, c_{i j} M_{j}\right\}\right. \\
& \left.+\sum_{j=1}^{n} \min \left\{d_{i j} m_{j}, d_{i j} M_{j}\right\}-I_{i}\right]>0 .
\end{aligned}
$$

We also can find some $t^{*} \geq 0$ so that $x_{i}\left(t^{*}\right)=E_{i}$ and obtain the following inequality:

$$
\begin{aligned}
\left.\frac{\mathrm{d} x_{i}(t)}{\mathrm{d} t}\right|_{t=t^{*}} \leq & a_{i}\left(E_{i}\right)\left[-b_{i}\left(E_{i}\right)+c_{i i} g_{i}\left(-E_{i}\right)+\sum_{\substack{j=1 \\
j \neq i}}^{n} \max \left\{c_{i j} m_{j}, c_{i j} M_{j}\right\}\right. \\
& \left.+\sum_{j=1}^{n} \max \left\{d_{i j} m_{j}, d_{i j} M_{j}\right\}-I_{i}\right] \\
< & 0 .
\end{aligned}
$$

From (8)-(11), we see that $x_{i}(t)$ would not escape from $\left[-E_{i}, p_{i}\right]$ when $i \in N_{1}^{\prime}$, and $x_{i}(t)$ would remain in $\left[q_{i}, E_{i}\right]$ when $i \in N_{3}^{\prime}$. Let $i$ go through $1,2, \ldots, n$, we get $x(t)$ is located in $\Omega_{1}$. In view of $\Omega_{1} \subseteq \Phi_{1}$, it implies that $\prod_{i=1}^{n} w_{i} \in \Phi_{1}$ is an invariant set of model (1) with the initial condition $x_{i}(s)=\varphi_{i}(s), s \in(-\infty, 0]$.

Denote $u_{i}(t)=x_{i}(t)-x_{i}^{\star}, i=1,2, \ldots, n$, then we have

$$
\begin{aligned}
\frac{\mathrm{d} u_{i}(t)}{\mathrm{d} t}= & -a_{i}\left(x_{i}(t)\right)\left[\left(b_{i}\left(x_{i}(t)\right)-b_{i}\left(x_{i}^{\star}\right)\right)\right. \\
& \left.-\sum_{j=1}^{n} c_{i j}\left(g_{j}\left(x_{j}(t)\right)-g_{j}\left(x_{j}^{\star}\right)\right)-\sum_{j=1}^{n} d_{i j}\left(f_{j}\left(x_{j}(t-\tau(t))\right)-f_{j}\left(x_{j}^{\star}\right)\right)\right] .
\end{aligned}
$$

Let $U_{i}(t)=\mu(t) u_{i}(t)$ and $U(t)=\sup _{s \leq t}\left(\max _{i=1,2, \ldots, n}\left(\zeta_{i}^{-1}\left|U_{i}(s)\right|\right)\right), t \geq T \geq T^{*}$. Then, for any $t^{*}, t^{*} \geq T \geq T^{*}$, we have $\max _{i=1,2, \ldots, n}\left(\zeta_{i}^{-1}\left|U_{i}\left(t^{*}\right)\right|\right) \leq U\left(t^{*}\right)$, which implies that $U(t)$ is bounded.

Let $i_{t^{*}}=i_{t^{*}}\left(t^{*}\right)$ when $\max _{i=1,2, \ldots, n}\left(\zeta_{i}^{-1}\left|U_{i}\left(t^{*}\right)\right|\right)=U\left(t^{*}\right)$ holds. Differentiating $\left|U_{i_{t^{*}}}(t)\right|$ at time $t^{*}$, we can deduce that

$$
\begin{aligned}
\left.\frac{\mathrm{d}\left|U_{i_{t^{*}}}(t)\right|}{\mathrm{d} t}\right|_{t=t^{*}}= & \operatorname{sign}\left(U_{i_{t^{*}}}\left(t^{*}\right)\right) \dot{\mu}\left(t^{*}\right) u_{i_{t^{*}}}\left(t^{*}\right)+\operatorname{sign}\left(U_{i_{t^{*}}}\left(t^{*}\right)\right) \mu\left(t^{*}\right) \\
& \cdot\left\{-a_{i_{t^{*}}}\left(x_{i_{t^{*}}}\left(t^{*}\right)\right)\left[\left(b_{i_{t^{*}}}\left(x_{i_{t^{*}}}\left(t^{*}\right)\right)-b_{i_{t^{*}}}\left(x_{i_{t^{*}}}^{\star}\right)\right)\right.\right.
\end{aligned}
$$




$$
\begin{aligned}
& -\sum_{j=1}^{n} c_{i_{t^{*}}}\left(g_{j}\left(x_{j}\left(t^{*}\right)\right)-g_{j}\left(x_{j}^{\star}\right)\right) \\
& \left.\left.-\sum_{j=1}^{n} d_{i_{t^{*}} j}\left(f_{j}\left(x_{j}\left(t^{*}-\tau\left(t^{*}\right)\right)\right)-f_{j}\left(x_{j}^{\star}\right)\right)\right]\right\} .
\end{aligned}
$$

By hypothesis (H2), there exists a positive constant $\beta_{i}$, so that

$$
b_{i}\left(x_{i}\right)-b_{i}\left(y_{i}\right)=\beta_{i}\left(x_{i}-y_{i}\right),
$$

where $\beta_{i} \geq b_{i}, i=1,2, \ldots, n$. Substituting (5), (6), (14) into (13) and applying (H1) and (H3), we can obtain the following inequality.

$$
\begin{aligned}
\left.\frac{\mathrm{d}\left|U_{i_{t^{*}}}(t)\right|}{\mathrm{d} t}\right|_{t=t^{*}}= & \operatorname{sign}\left(U_{i_{t^{*}}}\left(t^{*}\right)\right) \dot{\mu}\left(t^{*}\right) u_{i_{t^{*}}}\left(t^{*}\right)+\operatorname{sign}\left(U_{i_{t^{*}}}\left(t^{*}\right)\right) \mu\left(t^{*}\right) \\
& \cdot\left\{-a_{i_{t^{*}}}\left(x_{i_{t^{*}}}\left(t^{*}\right)\right)\left[\left(b_{i_{t^{*}}}\left(x_{i_{t^{*}}}\left(t^{*}\right)\right)-b_{i_{t^{*}}}\left(x_{i_{t^{*}}}^{*}\right)\right)\right.\right. \\
& -\sum_{j=1}^{n} c_{i_{t^{*}} j}\left(g_{j}\left(x_{j}\left(t^{*}\right)\right)-g_{j}\left(x_{j}^{*}\right)\right) \\
& \left.\left.-\sum_{j=1}^{n} d_{i_{t^{*}} j}\left(f_{j}\left(x_{j}\left(t^{*}-\tau\left(t^{*}\right)\right)\right)-f_{j}\left(x_{j}^{*}\right)\right)\right]\right\} \\
\leq & \left(-\underline{a}_{i_{t^{*}}} \beta_{i_{t^{*}}}+\frac{\dot{\mu}\left(t^{*}\right)}{\mu\left(t^{*}\right)}\right)\left|U_{i_{t^{*}}}\left(t^{*}\right)\right|+\sum_{j \in N_{1}} \bar{a}_{i_{t^{*}}}\left|c_{i_{t^{*}}}\right| \bar{\sigma}_{j}^{l}\left|U_{j}\left(t^{*}\right)\right| \\
& +\sum_{j \in N_{3}} \bar{a}_{i_{t^{*}}}\left|c_{i_{t^{*}} j}\right| \bar{\sigma}_{j}^{r}\left|U_{j}\left(t^{*}\right)\right| \\
& +\sum_{j \in N_{1}} \bar{a}_{i_{t^{*}}} \frac{\mu\left(t^{*}\right)}{\mu\left(t^{*}-\tau\left(t^{*}\right)\right)}\left|d_{i_{t^{*}} j}\right| \bar{\delta}_{j}^{l}\left|U_{j}\left(t^{*}-\tau\left(t^{*}\right)\right)\right| \\
& +\sum_{j \in N_{3}} \bar{a}_{i_{t^{*}}} \frac{\mu\left(t^{*}\right)}{\mu\left(t^{*}-\tau\left(t^{*}\right)\right)}\left|d_{i_{t^{*}} j}\right| \delta_{j}^{r}\left|U_{j}\left(t^{*}-\tau\left(t^{*}\right)\right)\right| \\
\leq & \left\{\left(-\underline{a}_{i_{t^{*}}} \beta_{i_{t^{*}}}+\frac{\dot{\mu}\left(t^{*}\right)}{\mu\left(t^{*}\right)}\right) \zeta_{i_{t^{*}}}+\sum_{j \in N_{1}} \bar{a}_{i_{t^{*}}}\left|c_{i_{t^{*}} j}\right| \bar{\sigma}_{j}^{l} \zeta_{j}+\sum_{j \in N_{3}} \bar{a}_{i_{t^{*}}}\left|c_{i_{t^{*}} j}\right| \bar{\sigma}_{j}^{r} \zeta_{j}\right. \\
& \left.+\frac{\mu\left(t^{*}\right)}{\mu\left(t^{*}-\tau\left(t^{*}\right)\right)}\left(\sum_{j \in N_{1}} \bar{a}_{i_{t^{*}}}\left|d_{i_{t^{*}} j}\right| \bar{\delta}_{j}^{l} \zeta_{j}+\sum_{j \in N_{3}} \bar{a}_{i_{t^{*}}}\left|d_{i_{t^{*}}}\right| \bar{\delta}_{j}^{r} \zeta_{j}\right)\right\} \cdot U\left(t^{*}\right) \\
< & 0 . \\
&
\end{aligned}
$$

From (15), we can see that there exists a positive constant $\delta_{1}$ such that $U(t)=U\left(t^{*}\right)$ for $t \in\left(t^{*}, t^{*}+\delta_{1}\right)$. Because of the arbitrariness of $t^{*}$, we can get that $U(t)=U(T)$ for all $t \geq$ $T \geq T^{*}$, which implies $\zeta_{i}^{-1}\left|\mu(t)\left(x_{i}(t)-x_{i}^{\star}\right)\right|<\max _{i=1,2, \ldots, n}\left(\zeta_{i}^{-1}\left|U_{i}(t)\right|\right)<U(t)$. Therefore, by Definition 1, the equilibrium $x^{\star}$ is $\mu$-stable in $\prod_{i=1}^{n} w_{i} \in \Phi_{1}$.

Next, we show that there exists an unstable equilibrium point in $\Phi_{2}$. 
Theorem 3 For any $\prod_{i=1}^{n} w_{i} \in \Phi_{2}$, given that (3) holds. If there are positive constants $\xi_{1}, \ldots, \xi_{n}$ such that

$$
\begin{gathered}
\min _{i \in N_{2}}\left\{\left(-\beta_{i}+c_{i i} \sigma_{i}^{m^{*}}\right) \xi_{i}-\sum_{j \in N_{1}} \xi_{j}\left|c_{i j}\right| \bar{\sigma}_{j}^{l}-\sum_{j \in N_{2}} \xi_{j}\left|c_{i j}\right| \bar{\sigma}_{j}^{m}-\sum_{j \in N_{3}} \xi_{j}\left|c_{i j}\right| \bar{\sigma}_{j}^{r}\right. \\
\left.-\sum_{j \in N_{1}} \xi_{j}\left|d_{i j}\right| \bar{\delta}_{j}^{l}-\sum_{j \in N_{2}} \xi_{j}\left|d_{i j}\right| \bar{\delta}_{j}^{m}-\sum_{j \in N_{3}} \xi_{j}\left|d_{i j}\right| \bar{\delta}_{j}^{r}\right\}>\max \{\lambda, 0\},
\end{gathered}
$$

where

$$
\begin{aligned}
\lambda \triangleq & \max _{i \in N_{1} \cup N_{3}}\left\{\left(-\beta_{i} \xi_{i}+\sum_{j \in N_{1}} \xi_{j}\left|c_{i j}\right| \bar{\sigma}_{j}^{l}+\sum_{j \in N_{2}} \xi_{j}\left|c_{i j}\right| \bar{\sigma}_{j}^{m}+\sum_{j \in N_{3}} \xi_{j}\left|c_{i j}\right| \bar{\sigma}_{j}^{r}\right.\right. \\
& \left.+\sum_{j \in N_{1}} \xi_{j}\left|d_{i j}\right| \bar{\delta}_{j}^{l}+\sum_{j \in N_{2}} \xi_{j}\left|d_{i j}\right| \bar{\delta}_{j}^{m}+\sum_{j \in N_{3}} \xi_{j}\left|d_{i j}\right| \bar{\delta}_{j}^{r}\right\}
\end{aligned}
$$

then the equilibrium point $x^{\star}$ of model (1) in $\prod_{i=1}^{n} w_{i} \in \Phi_{2}$ is unstable.

Proof Let $x(t)=\left(x_{1}(t), x_{2}(t), \ldots, x_{n}(t)\right)$ be an arbitrary solution of model (1) with the initial condition $x(s)=\varphi(s) \in \Phi_{2}, s \in(-\infty, 0]$, and let $u_{i}(t)=x_{i}(t)-x_{i}^{\star}, i=1,2, \ldots, n, t \geq 0$, where $x^{\star}=\left(x_{1}^{\star}, \ldots, x_{n}^{\star}\right)$ is one equilibrium point of model (1) in $\prod_{i=1}^{n} w_{i} \in \Phi_{2}$. Without loss of generality, suppose that $x(t)$ remains in $\prod_{i=1}^{n} w_{i} \in \Phi_{2}$. And we define $H(t)=$ $\sup _{t-\tau(t) \leq s \leq t}\left\{\max _{i=1,2, \ldots, n} \xi_{i}^{-1}\left|u_{i}(s)\right|\right\}, t \geq 0$. If $\max _{i \in N_{2}} \xi_{i}^{-1}\left|u_{i}(t)\right|=H(t)$ holds, denote a sequence item $i_{t}$ of $N_{2}$ such that $\xi_{i_{t}}^{-1}\left|u_{i_{t}}(t)\right|=\max _{i \in N_{2}} \xi_{i}^{-1}\left|u_{i}(t)\right|$, and differentiate $\left|u_{i_{t}}(t)\right|$ at time $t$, then we can deduce

$$
\begin{aligned}
\left.\frac{\mathrm{d}\left|u_{i_{t}}(t)\right|}{\mathrm{d} t}\right|_{(1)} & \operatorname{sign}\left(x_{i_{t}}(t)-x_{i_{t}}^{\star}\right) \cdot\left\{-a_{i_{t}}\left(x_{i_{t}}(t)\right)\left[\left(b_{i_{t}}\left(x_{i_{t}}(t)\right)-b_{i_{t}}\left(x_{i_{t}}^{\star}\right)\right)\right.\right. \\
& \left.\left.-\sum_{j=1}^{n} c_{i_{t}}\left(g_{j}\left(x_{j}(t)\right)-g_{j}\left(x_{j}^{\star}\right)\right)-\sum_{j=1}^{n} d_{i_{t}}\left(f_{j}\left(x_{j}\left(t-\tau_{i j}(t)\right)\right)-f_{j}\left(x_{j}^{\star}\right)\right)\right]\right\} .
\end{aligned}
$$

On account of hypothesis (H3), that is, $0 \leq \underline{\sigma}_{i}^{m} \leq \frac{g_{i}(u)-g_{i}(v)}{u-v} \leq \bar{\sigma}_{i}^{m}, \forall u, v \in\left[p_{i}, q_{i}\right]$, we can find a positive constant $\sigma_{i}^{m^{*}}$ such that

$$
\frac{g_{i}(u)-g_{i}(v)}{u-v}=\sigma_{i}^{m^{*}}
$$

Therefore by (14), (18), hypothesis (H1) and (H3), Eq. (17) can be further converted to

$$
\begin{aligned}
\left.\frac{\mathrm{d}\left|u_{i_{t}}(t)\right|}{\mathrm{d} t}\right|_{(1)}= & \left\{a _ { i _ { t } } ( x _ { i _ { t } } ( t ) ) \left[-\operatorname{sign}\left(u_{i_{t}}(t)\right) \cdot \beta_{i_{t}} \cdot u_{i_{t}}(t)+\operatorname{sign}\left(u_{i_{t}}(t)\right) \cdot c_{i_{t} i_{t}}\left(\sigma_{i}^{m^{*}} \cdot u_{i_{t}}(t)\right)\right.\right. \\
& +\operatorname{sign}\left(u_{i_{t}}(t)\right) \cdot \sum_{\substack{j=1 \\
j \neq i_{t}}}^{n} c_{i_{t} j}\left(g_{j}\left(u_{j}(t)+x_{j}^{\star}\right)-g_{j}\left(x_{j}^{\star}\right)\right) \\
& \left.\left.+\operatorname{sign}\left(u_{i_{t}}(t)\right) \cdot \sum_{j=1}^{n} d_{i_{t} j}\left(f_{j}\left(u_{j}\left(t-\tau_{i j}(t)\right)+x_{j}^{\star}\right)-f_{j}\left(x_{j}^{\star}\right)\right)\right]\right\}
\end{aligned}
$$




$$
\begin{aligned}
\geq & \left\{a _ { i _ { t } } ( x _ { i _ { t } } ( t ) ) \left[\left(-\beta_{i_{t}}+c_{i_{t} i_{t}} \sigma_{i}^{m^{*}}\right)\left|u_{i_{t}}(t)\right|-\sum_{j \in N_{1}}\left|c_{i_{t} j}\right| \bar{\sigma}_{j}^{l}\left|u_{j}(t)\right|\right.\right. \\
& -\sum_{\substack{j \in N_{2} \\
j \neq i i_{t}}}\left|c_{i_{t} j}\right| \bar{\sigma}_{j}^{m}\left|u_{j}(t)\right|-\sum_{j \in N_{3}}\left|c_{i_{t} j}\right| \bar{\sigma}_{j}^{r}\left|u_{j}(t)\right|-\sum_{j \in N_{1}}\left|d_{i_{t} j}\right| \bar{\delta}_{j}^{l}\left|u_{j}\left(t-\tau_{i j}(t)\right)\right| \\
& \left.\left.-\sum_{j \in N_{2}}\left|d_{i_{t} j}\right| \bar{\delta}_{j}^{m}\left|u_{j}\left(t-\tau_{i j}(t)\right)\right|-\sum_{j \in N_{3}}\left|d_{i_{t}}\right| \bar{\delta}_{j}^{r}\left|u_{j}\left(t-\tau_{i j}(t)\right)\right|\right]\right\} \\
\geq & a_{i_{t}}\left(x_{i_{t}}(t)\right)\left\{\left(-\beta_{i_{t}}+c_{i_{t} i_{t}} \sigma_{i}^{m^{*}}\right) \xi_{i_{t}}-\left[\sum_{\substack{j \in N_{j} \\
j \neq i_{t}}} \xi_{j}\left|c_{i_{t} j}\right| \bar{\sigma}_{j}^{m}+\sum_{j \in N_{2}} \xi_{j}\left|d_{i_{t} j}\right| \bar{\delta}_{j}^{m}\right]\right. \\
& \left.-\left[\sum_{j \in N_{1}} \xi_{j}\left|c_{i_{t} j}\right| \bar{\sigma}_{j}^{l}+\sum_{j \in N_{3}} \xi_{j}\left|c_{i_{t} j}\right| \bar{\sigma}_{j}^{r}+\sum_{j \in N_{1}} \xi_{j}\left|d_{i_{t}}\right| \bar{\delta}_{j}^{l}+\sum_{j \in N_{3}} \xi_{j}\left|d_{i_{t} j}\right| \bar{\delta}_{j}^{r}\right]\right\} H(t) \\
> & a_{i_{t}}\left(x_{i_{t}}(t)\right) \max \{\lambda, 0\} H(t) .
\end{aligned}
$$

Inequality (19) implies that there exists a number $r>0$ such that $\left|u_{i t}(s)\right|>\left|u_{i t}(t)\right|, s \in(t$, $t+r)$.

Besides, suppose that there exists some time point $t^{\prime}$ such that

$$
\begin{aligned}
& \sup _{t^{\prime}-\tau\left(t^{\prime}\right) \leq s \leq t^{\prime}}\left\{\max _{i \in N_{1} \cup N_{3}} \xi_{i}^{-1}\left|u_{i}(s)\right|\right\}=\sup _{t^{\prime}-\tau\left(t^{\prime}\right) \leq s \leq t^{\prime}}\left\{\max _{i \in N_{2}} \xi_{i}^{-1}\left|u_{i}(s)\right|\right\}, \\
& \sup _{t^{\prime}-\tau\left(t^{\prime}\right) \leq s \leq t^{\prime}}\left\{\max _{i \in N_{1} \cup N_{3}} \xi_{i}^{-1}\left|u_{i}(s)\right|\right\}=\max _{i \in N_{1} \cup N_{3}} \xi_{i}^{-1}\left|u_{i}\left(t^{\prime}\right)\right|,
\end{aligned}
$$

and denote a sequence item $i^{\prime} \in N_{1} \cup N_{3}$ such that $\xi_{i^{\prime}}^{-1}\left|u_{i^{\prime}}\left(t^{\prime}\right)\right|=\max _{i \in N_{1} \cup N_{3}} \xi_{i}^{-1}\left|u_{i}\left(t^{\prime}\right)\right|$. Hence we can get

$$
\begin{aligned}
\left.\frac{\mathrm{d}\left|u_{i^{\prime}}(t)\right|}{\mathrm{d} t}\right|_{t=t^{\prime}}= & \left\{a _ { i ^ { \prime } } ( x _ { i ^ { \prime } } ( t ^ { \prime } ) ) \left[-\operatorname{sign}\left(u_{i^{\prime}}(t)\right) \cdot \beta_{i^{\prime}} \cdot u_{i^{\prime}}\left(t^{\prime}\right)\right.\right. \\
& +\operatorname{sign}\left(u_{i^{\prime}}\left(t^{\prime}\right)\right) \cdot \sum_{j=1}^{n} c_{i^{\prime} j}\left(g_{j}\left(u_{j}\left(t^{\prime}\right)+x_{j}^{r}\right)-g_{j}\left(x_{j}^{r}\right)\right) \\
& \left.\left.+\operatorname{sign}\left(u_{i^{\prime}}\left(t^{\prime}\right)\right) \cdot \sum_{j=1}^{n} d_{i^{\prime} j}\left(f_{j}\left(u_{j}\left(t-\tau_{i^{\prime} j}(t)\right)+x_{j}^{r}\right)-f_{j}\left(x_{j}^{r}\right)\right)\right]\right\} \\
\leq & \left\{a _ { i ^ { \prime } } ( x _ { i ^ { \prime } } ( t ^ { \prime } ) ) \left[-\beta_{i^{\prime}}\left|u_{i^{\prime}}\left(t^{\prime}\right)\right|+\sum_{j \in N_{1}}\left|c_{i^{\prime}}\right| \bar{\sigma}_{j}^{l}\left|u_{j}\left(t^{\prime}\right)\right|+\sum_{j \in N_{2}}\left|c_{i^{\prime} j}\right| \bar{\sigma}_{j}^{m}\left|u_{j}\left(t^{\prime}\right)\right|\right.\right. \\
& +\sum_{j \in N_{3}}\left|c_{i^{\prime} j}\right| \bar{\sigma}_{j}^{r}\left|u_{j}\left(t^{\prime}\right)\right|+\sum_{j \in N_{1}}\left|d_{i^{\prime} j}\right| \bar{\delta}_{j}^{l}\left|u_{j}\left(t^{\prime}-\tau_{i^{\prime} j}\left(t^{\prime}\right)\right)\right| \\
& \left.\left.+\sum_{j \in N_{2}}\left|d_{i^{\prime} j}\right| \bar{\delta}_{j}^{m}\left|u_{j}\left(t^{\prime}-\tau_{i^{\prime} j}\left(t^{\prime}\right)\right)\right|+\sum_{j \in N_{3}}\left|d_{i^{\prime}} j\right| \bar{\delta}_{j}^{r}\left|u_{j}\left(t^{\prime}-\tau_{i j}\left(t^{\prime}\right)\right)\right|\right]\right\} \\
\leq & \left\{a _ { i ^ { \prime } } ( x _ { i ^ { \prime } } ( t ^ { \prime } ) ) \left[\left(-\beta_{i^{\prime}} \xi_{i^{\prime}}+\sum_{j \in N_{2}} \xi_{j}\left|c_{i^{\prime}} j\right| \bar{\sigma}_{j}^{m}+\sum_{j \in N_{2}} \xi_{j}\left|d_{i^{\prime} j}\right| \bar{\delta}_{j}^{m}+\sum_{j \in N_{1}} \xi_{j}\left|c_{i^{\prime} j}\right| \bar{\sigma}_{j}^{l}\right.\right.\right. \\
& \left.\left.+\sum_{j \in N_{3}} \xi_{j}\left|c_{i^{\prime} j}\right| \bar{\sigma}_{j}^{r}+\sum_{j \in N_{1}} \xi_{j}\left|d_{i^{\prime} j}\right| \bar{\delta}_{j}^{l}+\sum_{j \in N_{3}} \xi_{j}\left|d_{i^{\prime}} j\right| \bar{\delta}_{j}^{r}\right]\right\} H\left(t^{\prime}\right) \\
\leq & a_{i^{\prime}}\left(x_{i^{\prime}}\left(t^{\prime}\right)\right) \lambda H\left(t^{\prime}\right) . \\
&
\end{aligned}
$$


In the meantime, we can obtain the derivative of $\left|u_{i^{\prime} t^{\prime}}(t)\right|$ at time $t^{\prime}$ by (19) when $i^{\prime} \in N_{2}$

$$
\left.\frac{\mathrm{d}\left|u_{i^{\prime} t^{\prime}}(t)\right|}{\mathrm{d} t}\right|_{t=t^{\prime}}>a_{i^{\prime} t^{\prime}}\left(x_{i^{\prime} t^{\prime}}\left(t^{\prime}\right)\right) \max \{\lambda, 0\} H\left(t^{\prime}\right)
$$

where $\xi_{i^{\prime} t^{\prime}}^{-1}\left|u_{i^{\prime} t^{\prime}}\left(t^{\prime}\right)\right|=\max _{i^{\prime} \in N_{2}} \xi_{i^{\prime}}^{-1}\left|u_{i^{\prime}}\left(t^{\prime}\right)\right|$.

Given the above, we can conclude that

$$
H(t)=\sup _{t-\tau(t) \leq s \leq t}\left\{\max _{i \in N_{2}} \xi_{i}^{-1}\left|u_{i}(s)\right|\right\} \geq \sup _{-\tau(t) \leq s \leq 0}\left\{\max _{i \in N_{2}} \xi_{i}^{-1}\left|u_{i}(s)\right|\right\}
$$

holds for all $t \geq 0$, and there exists an increasing time sequence $\left\{t_{l}\right\}_{l=1}^{\infty}$ with $\lim _{l \rightarrow \infty} t_{l}=$ $+\infty$ such that $\sup _{t_{l}-\tau\left(t_{l}\right) \leq s \leq t_{l}}\left\{\max _{i \in N_{2}} \xi_{i}^{-1}\left|u_{i}(s)\right|\right\}=\max _{i \in N_{2}} \xi_{i}^{-1}\left|u_{i}\left(t_{l}\right)\right|$. Correspondingly, there exists an increasing time subsequence $\left\{t_{l_{k}}\right\}_{k=1}^{\infty}$ with $\lim _{k \rightarrow \infty} t_{l_{k}}=+\infty$ such that $\xi_{i^{\prime \prime}}^{-1}\left|u_{i^{\prime \prime}}\left(t_{l_{k}}\right)\right|=\sup _{t_{l_{k}}-\tau\left(t_{l_{k}}\right) \leq s \leq t_{l_{k}}}\left\{\max _{i \in N_{2}} \xi_{i}^{-1}\left|u_{i}(s)\right|\right\} \geq \sup _{-\tau(t) \leq s \leq 0}\left\{\max _{i \in N_{2}} \xi_{i}^{-1}\left|u_{i}(s)\right|\right\}>0$, $k=1,2, \ldots$. Thus $u_{i^{\prime \prime}}(t)$ would not converge to 0 when $t \rightarrow+\infty$ means that the equilibrium point $x^{\star}$ is unstable in $\prod_{i=1}^{n} w_{i} \in \Phi_{2}$.

\section{Corollaries and comparison}

According to the above theorems, we have the following two corollaries.

Corollary 1 If conditions (3)-(6) and (16) hold, then model (1) has at least $3^{n}$ equilibrium points in $R^{n}, 2^{n}$ of which are locally $\mu$-stable in $\Phi_{1}$, the remaining $3^{n}-2^{n}$ points are unstable in $\Phi_{2}$.

Corollary 2 When $a_{i}\left(x_{i}(t)\right)=1$, model (1) becomes the Hopfield neural network model

$$
\frac{\mathrm{d} x_{i}(t)}{\mathrm{d} t}=-b_{i}\left(x_{i}(t)\right)+\sum_{j=1}^{n} c_{i j} g_{j}\left(x_{j}(t)\right)+\sum_{j=1}^{n} d_{i j} f_{j}\left(x_{j}(t-\tau(t))\right)-I_{i}, \quad t \geq 0 .
$$

On the basis of conditions (3)-(6) and (16), model (22) has at least $3^{n}$ equilibrium points in $R^{n}, 2^{n}$ of which are locally $\mu$-stable in $\Phi_{1}$, the remaining $3^{n}-2^{n}$ points are unstable equilibrium points in $\Phi_{2}$.

Remark 1 The present paper investigates the multistability of a Cohen-Grossberg neural network with unbounded time-varying delay and nondecreasing activation functions. Compared with [41], the obtained results are more general.

Remark 2 The net self-inhibition function $b_{i}\left(x_{i}(t)\right)$ in this paper is an odd function and monotonically increasing, which includes the case of [41]. Thus model (22) is more general than [41].

Remark 3 The activation functions of [41] are identical whether with or without time delay, but the activation functions in this paper are different. Therefore the conclusion of this paper is closer to the practical application. 

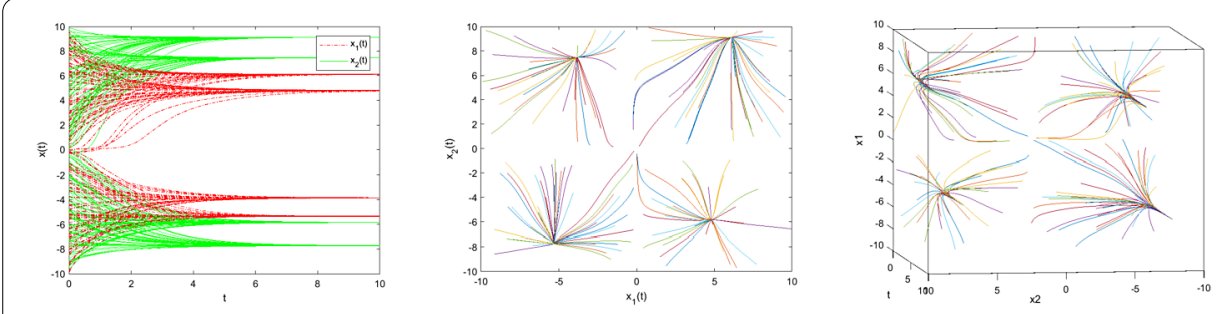

Figure 1 The state trajectories and phase plots of the neural network model (1). The above three graphs are the state trajectories and phrase plots, where the third graph is a $3 D$ phase plot of the state variable $\left(t, x_{2}, x_{1}\right)$

\section{Simulation example}

Example We consider the following two-dimensional Cohen-Grossberg neural network model:

$$
\frac{\mathrm{d} x_{i}(t)}{\mathrm{d} t}=-a_{i}\left(x_{i}(t)\right)\left[b_{i}\left(x_{i}(t)\right)-\sum_{j=1}^{n} c_{i j} g_{j}\left(x_{j}(t)\right)-\sum_{j=1}^{n} d_{i j} f_{j}\left(x_{j}\left(t-\tau_{i j}(t)\right)\right)+I_{i}(t)\right], \quad t \geq 0,
$$

where $i=1,2, a(x)=1+0.2 \sin (x), b_{1}\left(x_{1}(t)\right)=x_{1}(t), b_{2}\left(x_{2}(t)\right)=-1.2 x_{2}(t)$,

$$
\begin{aligned}
& g(x)= \begin{cases}\tanh (0.2 x)-\tanh (1)+\tanh (0.2), & x<-1, \\
\tanh (x), & -1 \leq x \leq 1, \\
\tanh (0.2 x)+\tanh (1)-\tanh (0.2), & x>1,\end{cases} \\
& C=\left(c_{i j}\right)=\left(\begin{array}{ll}
3.5 & 0.2 \\
0.4 & 4.8
\end{array}\right), \quad D=\left(d_{i j}\right)=\left(\begin{array}{ll}
0.4 & 0.2 \\
0.2 & 0.5
\end{array}\right), \quad I=\left(\begin{array}{l}
-0.3 \\
-0.6
\end{array}\right), \\
& \tau_{i j}(t)=\tau(t)=0.2 t .
\end{aligned}
$$

After a simple calculation, we know that the above hypothesis satisfies the conditions of Theorems $1-3$. Therefore there are at least nine equilibrium points in model (1) from Corollary 1,4 of which are $\mu$-stable equilibrium points and others are unstable points. The solution of model (1) is traced with 150 initial conditions, the simulation results are showed in the above three graphs of Fig. 1.

\section{Conclusion}

Stability of multiple unstable Cohen-Grossberg neural networks with unbounded timevarying delays is discussed analytically in this paper. Based on the geometric structure of two different activation functions and some rigorous mathematical analysis, the present paper proved that there exist multiple equilibrium points in the model, some of which are unstable, others are $\mu$-stable. One numerical example and its simulation show the effectiveness of the conclusion. Here, we also need point out the following. On the one hand, the impulsive control is rarely used to deal with cases of unbounded time-varying delays, especially for multiple unstable Cohen-Grossberg neural networks with unbounded time-varying delays. Therefore, the stability under impulsive control of multiple unstable Cohen-Grossberg neural networks with unbounded time-varying delays is still a challenging problem. On the other hand, we use something like positivity-based method to 
study the stability of Cohen-Grossberg neural networks in this article. The positivitybased method is a valid approach for difference and delay differential systems (see [24-28, 44-48]). Therefore, the research on stability with positivity-based approach is an interesting and meaningful topic, and we will also consider the stability of other neural networks by employing the positivity-based approach in the near future.

\author{
Acknowledgements \\ The authors would like to thank the referees for their valuable comments and suggestion. \\ Funding \\ This work was supported in part by the Scientific and Technological Research Program of Chongqing Municipal \\ Education Commission (Grant No. KJ1710254), the Foundation of Chongqing Three Gorges University (Grant \\ No. 14ZD16), Chongqing Municipal Key Laboratory of Institutions of Higher Education (Grant No. [2017]3), Program of \\ Chongqing Development and Reform Commission (Grant No. 2017[1007]).
}

\title{
Availability of data and materials
}

Not applicable.

\section{Competing interests}

The authors declare that they have no competing interests.

\section{Authors' contributions}

The authors contributed equally to the writing of this paper. The authors read and approved the final manuscript.

\section{Publisher's Note}

Springer Nature remains neutral with regard to jurisdictional claims in published maps and institutional affiliations.

Received: 3 December 2018 Accepted: 7 June 2019 Published online: 21 June 2019

\section{References}

1. Cohen, M.A., Grossberg, S.: Absolute stability of global pattern formation and parallel memory storage by competitive neural networks. IEEE Trans. Circuits Syst. I 42, 815-826 (1983)

2. Lu, W., Chen, T: New conditions on global stability of Cohen-Grossberg neural networks. Neural Comput. 15 $1173-1189(2003)$

3. Liao, X., Li, C., Wong, K.: Criteria for exponential stability of Cohen-Grossberg neural networks. Neural Netw. 17, 1401-1414 (2004)

4. Rong, L.: LMI-based criteria for robust stability of Cohen-Grossberg neural networks with delay. Phys. Lett. A 339(1-2), 63-73 (2005)

5. Huang, C., Huang, L.: Dynamics of a class of Cohen-Grossberg neural networks with time-varying delays. Nonlinear Anal., Real World Appl. 8, 40-52 (2007)

6. Yuan, K., Cao, J.: An analysis of global asymptotic stability of delayed Cohen-Grossberg neural networks via nonsmooth analysis. IEEE Trans. Circuits Syst. I 52, 1854-1861 (2005)

7. Zhang, J., Suda, Y., Iwasa, T.: Absolutely exponential stability of Cohen-Grossberg neural networks with variable delays. Phys. Lett. A 338, 44-50 (2005)

8. Song, Q., Wang, Z:: Stability analysis of impulsive stochastic Cohen-Grossberg neural networks with mixed time delays. Physica A 387, 3314-3326 (2008)

9. Wang, Z., Liu, Y., Li, M., Liu, X.: Stability analysis for stochastic Cohen-Grossberg neural networks with mixed time delays. IEEE Trans. Neural Netw. 17, 814-820 (2006)

10. Yu, W., Cao, J., Wang, J.: An LMI approach to global asymptotic stability of the delayed Cohen-Grossberg neural network via nonsmooth analysis. Neural Netw. 20, 810-818 (2007)

11. Cao, J., Song, Q.: Stability in Cohen-Grossberg-type bidirectional associative memory neural networks with time-varying delays. Nonlinearity 19, 1601-1607 (2006)

12. Yang, F., Zhang, C., Wu, D.: Global stability analysis of impulsive BAM type Cohen-Grossberg neural networks with delays. Appl. Math. Comput. 186, 932-940 (2007)

13. Wang, Y., Cao, J., Huang, G.: LMI-based criteria for exponential robust stability of Cohen-Grossberg-type bidirectional associative memory neural networks with delays. Syst. Sci. Control Eng. 222, 57-67 (2008)

14. Jiang, H., Cao, J.: BAM-type Cohen-Grossberg neural networks with time delays. Math. Comput. Model. 47, 92-103 (2008)

15. Yang, Z., Huang, Y.: Exponential dissipativity of impulsive Cohen-Grossberg neural networks with mixed delays. J. Sichuan Univ. Natur. Sci. Ed. 3, 464-468 (2010)

16. Li, L., Jian, J.: Exponential convergence and Lagrange stability for impulsive Cohen-Grossberg neural networks with time-varying delays. J. Comput. Appl. Math. 277, 23-35 (2015)

17. Hu, J., Zeng, C.: Adaptive exponential synchronization of complex-valued Cohen-Grossberg neural networks with known and unknown parameters. Neural Netw. 86, 90-101 (2017)

18. Xu, X., Song, Q., Zhang, J., Shi, J., Zhao, L.: Dynamical behavior analysis of a class of complex-valued Cohen-Grossberg neural networks with time-varying delays. Appl. Math. Mech. 12, 1389-1398 (2017) 
19. Tan, M.: Stabilization of coupled time-delay neural networks with nodes of different dimensions. Neural Process. Lett. 43(1), 255-268 (2016)

20. Shu, H., Song, Q., Liu, Y., Zhao, Z., Alsaadi, F.E.: Global $\mu$-stability of quaternion-valued neural networks with non-differentiable time-varying delays. Neurocomputing 247, 202-212 (2017)

21. Chen, T., Liu, X:: $\mu$-Stability of nonlinear positive systems with unbounded time-varying delays. IEEE Trans. Neural Netw. Learn. Syst. 28(7), 1710-1715 (2017)

22. Tu, Z., Cao, J., Hayat, T.: Global exponential stability in Lagrange sense for inertial neural networks with time-varying delays. Neurocomputing 171, 524-531 (2016)

23. Park, M.J., Kwon, O.M., Choi, S.G.: Stability analysis of discrete-time switched systems with time-varying delays via a new summation inequality. Nonlinear Anal. Hybrid Syst. 23, 76-90 (2017)

24. Ngoc, P.H.A.: Stability of positive differential systems with delay. IEEE Trans. Autom. Control 58(1), 203-209 (2013)

25. Domoshnitsky, A., Fridman, E.: A positivity-based approach to delay-dependent stability of systems with large time-varying delays. Syst. Control Lett. 97, 139-148 (2016)

26. Domoshnitsky, A., Goltser, Ya.: Approach to study of stability and bifurcation of integro-differential equations. Math. Comput. Model. 36, 663-678 (2002)

27. Agarwal, R.P., Bohner, M., Domoshnitsky, A., Goltser, Y.: Floquet theory and stability of nonlinear integro-differential equations. Acta Math. Hung. 109(4), 305-330 (2005)

28. Bainov, D., Domoshnitsky, A.: Nonnegativity of the Cauchy matrix and exponential stability of a neutral type system of functional-differential equations. Extr. Math. 8(1), 75-82 (1993)

29. Kaslik, E., Sivasundaram, S.: Multistability in impulsive hybrid Hopfield neural networks with distributed delays. Nonlinear Anal., Real World Appl. 12, 1640-1649 (2011)

30. Liang, J., Gong, W., Huang, T:. Multistability of complex-valued neural networks with discontinuous activation functions. Neural Netw. 84, 125-142 (2016)

31. Nie, X., Zheng, W.: Multistability and instability of neural networks with discontinuous nonmonotonic piecewise linear activation functions. IEEE Trans. Neural Netw. Learn. Syst. 26(11), 2901-2913 (2015)

32. Nie, X., Zheng, W.: Multistability of neural networks with discontinuous non-monotonic piecewise linear activation functions and time-varying delays. Neural Netw. 65, 65-79 (2015)

33. Huang, G., Cao, J: Delay-dependent multistability in recurrent neural networks. Neural Netw. 23(2), 201-209 (2010)

34. Liu, P., Zeng, Z., Wang, J.: Multistability analysis of a general class of recurrent neural networks with non-monotonic activation functions and time-varying delays. Neural Netw. 79, 117-127 (2016)

35. Wang, L., Lu, W., Chen, T.: Coexistence and local stability of multiple equilibria in neural networks with piecewise linear nondecreasing activation functions. Neural Netw. 23, 189-200 (2010)

36. Yang, W., Wang, Y., Zeng, Z., Zheng, D.: Multistability of discrete-time delayed Cohen-Grossberg neural networks with second-order synaptic connectivity. Neurocomputing 164, 252-261 (2015)

37. Liu, P., Zeng, Z., Wang, J.: Multistability of recurrent neural networks with non-monotonic activation functions and mixed time delays. IEEE Trans. Syst. Man Cybern. 46(4), 512-523 (2016)

38. Chen, X., Zhao, Z., Song, Q., Hu, J.: Multistability of complex-valued neural networks with time-varying delays. Appl. Math. Comput. 294, 18-35 (2017)

39. Wang, L., Chen, T:: Multistability of neural networks with Mexican-hat-type activation functions. IEEE Trans. Neural Netw. Learn. Syst. 23(11), 1816-1826 (2012)

40. Du, Y., Li, Y., Xu, R.: Multistability and multiperiodicity for a general class of delayed Cohen-Grossberg neural networks with discontinuous activation functions. Discrete Dyn. Nat. Soc. 2013, 917835 (2013)

41. Wang, L., Chen, T:: Multiple $\mu$-stability of neural networks with unbounded time-varying delays. Neural Netw. 53 , 109-118 (2014)

42. Chen, X., Zhao, Z., Song, Q., Hu, J.: Multistability of complex-valued neural networks with time-varying delays. Appl. Math. Comput. 294, 18-35 (2017)

43. Tan, M., Xu, D.: Multiple $\boldsymbol{\mu}$-stability analysis for memristor-based complex-valued neural networks with nonmonotonic piecewise nonlinear activation functions and unbounded time-varying delays. Neurocomputing $275,2681-2701(2018)$

44. Smith, H.L.: Monotone Dynamical Systems: An Introduction to the Theory of Competitive and Cooperative Systems. American Mathematical Society, Providence (1995)

45. Farina, L., Rinaldi, S.: Positive Linear Systems: Theory and Applications. Wiley-Interscience, New York (2000)

46. Azbelev, N.V., Simonov, P.M.: Stability of Differential Equations with After Effect. Stability and Control: Theory, Methods and Applications, vol. 20. Taylor \& Francis, London (2003)

47. Haddad, W.M., Chellaboina, V., Hui, Q.: Nonnegative and Compartmental Dynamical Systems. Princeton University Press, Princeton (2010)

48. Agarwal, R.P., Berezansky, L., Braverman, E., Domoshnitsky, A.: Nonoscillation Theory of Functional Differential Equations with Applications. Springer, New York (2012) 